\title{
Soret effect on transient magnetohydrodynamic nanofluid flow past a vertical plate through a porous medium with second order chemical reaction and thermal radiation
}

\author{
Amit Kumar ${ }^{1 *}$, Ramayan Singh $^{1}$, Gauri Shanker Seth ${ }^{2}$, Rajat Tripathi ${ }^{1}$ \\ ${ }^{1}$ Department of Mathematics, National Institute of Technology, Jamshedpur 831014, Jharkhand, India \\ ${ }^{2}$ Indian Institute of Technology, Indian School of Mines, Dhanbad 826004, Jharkhand, India
}

Corresponding Author Email: rajat17mnnit@gmail.com

https://doi.org/10.18280/ijht.360435

Received: 3 January 2017

Accepted: 6 February 2018

\section{Keywords:}

nanofluid, Soret effect, MHD, chemical reaction, porous medium

\begin{abstract}
In the present article, an investigation on the impact of Soret effect on unsteady magnetohydrodynamic natural convection flow of a viscous, electrically conducting and incompressible nanofluid over a vertical plate through a medium filled with porous materials with the consideration of a second order chemical reaction, has been investigated. Three kinds of water-based nanofluids, comprising of aluminum oxide $\left(\mathrm{Al}_{2} \mathrm{O}_{3}\right)$, titanium oxide $\left(\mathrm{TiO}_{2}\right)$ and silver $(A g)$ as nanoparticles, are chosen for this analysis. Governing equations accompanied by the boundary and initial conditions are changed into non-dimensional form and then they are solved by Crank-Nicolson type finite difference scheme. The impact of relevant flow parameters on nanofluid velocity, nanofluid temperature and species concentration are depicted graphically with comprehensive discussions whereas numerical findings for coefficient of skin friction, wall temperature gradient i.e. Nusselt number and wall concentration gradient are depicted in tabular form. It has been observed that Soret effect has the ability to enhance the species concentration. The investigation we have performed here, has various scientific and industrial applications.
\end{abstract}

\section{INTRODUCTION}

The concept of nanofluids was proposed by Choi [1] at Argonne National Laboratory (ANL). Nanofluids are proved to be the promising fluids for the improvement of heat transfer because of their extraordinary thermal conductivity property, where nano-sized particles (minor than 100 nanometers) are suspended uniformly in a base fluid. The type of nanoparticle used is a kind of big deal as far as the enhancement of thermal conductivity of the base fluid is concerned. In the recent past, nanofluids are used extensively in numerous scientific processes such as transportation, power generation, treatment of cancer-infected tissues, electronic and space cooling, etc. Different nanosized particles of metals and their oxides such as $\mathrm{Cu}, \mathrm{Ag}, \mathrm{Al}_{2} \mathrm{O}_{3}$ and $\mathrm{CuO}$, carbides ( $\mathrm{SiC}$ ) or non-metals (graphite, carbon nanotubes) are frequently used as nanoparticles while preparing nanofluid. Selvakumar and Dhinakaran [2] studied the Krieger-Dougherty (K-D) model to describe the effective nanofluid viscosity based on PSD (particle size distribution) study. They analysed the effect of aggregation together with the interfacial layer formation in the $\mathrm{K}-\mathrm{D}$ model in order to make it a possible nanofluids. Heris et al. [3] investigated the laminar flow of $\mathrm{Al}_{2} \mathrm{O}_{3}$-nanofluid in a circular tube experimentally and showed that the coefficient of convective heat transfer of pure water was improved by $41 \%$ with only $3 \%$ volume fraction of $\mathrm{Al}_{2} \mathrm{O}_{3}$ nanoparticle. Fotukian and Esfahany [4] considered the influence of $\mathrm{CuO}$-nanofluid in a round tube in a turbulent flow situation and observed that the coefficient of convective heat transfer was boosted by $25 \%$ when the concentration of cupric oxide is taken low. In the recent few years, the impact of magnetohydrodynamic (MHD) nanofluid flows together with mass and heat transfer have attracted several researchers, engineers and scientists due to their numerous real world applications in contemporary technology and many industries such as in cooling of fission reactors, power generation in space craft, stellar and solar structures, medicine delivery processing, hypersonic wind tunnel experiments, liquid metals, MHD pumps and radio propagation. Inspired from such real-world applications, many researcher and engineers have carried out the research work on MHD, experimentally as well as theoretically. Turkyilmazoglu [5] analysed the flow of four different kinds of nanofluids viz. Cu-nanofluid, Ag-nanofluid, $\mathrm{TiO}_{2}$ nanofluid and $\mathrm{CuO}$-nanofluid and found the analytical solution for magnetohydrodynamic slip and mass transport in nanofluids. Sheikholeslami et al. [6] analyzed the flow of $\mathrm{CuO}$ - nanofluid under the effect of magnetic field and found the enhancements in heat transfer for higher values of magnetic parameter. Sandeep and Reddy [7] discussed MHD $\mathrm{Cu}$ nanofluid flow near a cone and a wedge and they observed that volume fraction of nanoparticle and magnetic parameter strengthen the temperature field.

While analyzing the free convection flow of hydromagnetic liquids to characterize the heat transfer behavior, the radiative property of the liquid cannot be overlooked as it has substantial influence on the heat transfer characteristic of flow. This radiative property of the liquid affects the heat transfer performance of several industrial and engineering processes such as furnace design, turbine blade heat transfer, polymer processing, solar energy collector, glass production, heating and cooling chambers etc. Considering all these applications of thermal radiation effect in real flow situations, several scientists and researchers continue to delve deeper into the study of the influence of thermal radiation. Takhar et al. [8] 
scrutinized the radiation effect on free convection flow of a hydromagnetic fluid over a semi-infinite vertical plate. Das and Jana [9] examined the MHD and thermal radiation effects on free convection flow of water based nanofluid with three kinds of nanoparticles, past an infinite moving horizontal plate. They deduced that a surge in the radiation corresponds to a deceleration in the fluid velocity. Some other important studies on the topic are due to Ogulu and Makinde [10], Mahmoud [11], Das et al. [12], Seth et al. [13-14].

It is observed that in all above experimental or theoretical studies, thermophoresis or Soret effect was ignored in mass transfer process because of their small order of magnitude. Thermophoresis or Soret effects are noteworthy when there exists a difference in the density in the flow field, as in the case of geo-sciences and hydrological fluid flow situation. Soret effect is observed in various flow situations such as, in a compound with medium molecular weight $\left(N_{2}\right)$ and of very light molecular weight $\left(\mathrm{H}_{2}\right)$, in isotope separation, etc. For different fluids, many scientists have studied the thermophoresis effect due to its significant applications in science. Considering the significance of such effect, Ahmed [15] analysed the Hartmann flow restricted by two parallel isothermal horizontal plates and presented the analytical solution with the consideration of Soret effect. Hussanan et al. [16] examined the MHD mixed-convection flow past an oscillating vertical plate, in consideration of thermophoresis effect and observed that the species concentration gets enhanced with an increment in Soret number. Seth et al. [17] discussed the Soret effect on transient MHD natural convection flow near a vertical moving plate with rampedness in the temperature of the plate, in a rotating system. They observed that thermophoresis effect has a tendency to distribute the species throughout the flow boundary layer and it decreases the concentration gradient at the plate. Zhao et al. [18] considered the free convection stream of hydromagnetic fractional viscoelastic fluid in a porous medium, taking Dufour and Soret effects into account. Some other related research work on this theme are also due to Reddy and Chamkha. [19], Saritha et al. [20], Bég et al. [21], Abdel-wahed and S. M. Abdel-AAL [22].

Chemical reaction effect on mass transfer flows are vital in a lot of industrial applications. Some of the industrial and technological applications of chemical reactions are observed in chemical industries viz. polymer production, manufacture of ceramics, food processing, catalytic chemical reactors, hydrometallurgical industries, polymer production etc. Due to the significance of reaction of chemicals in all sorts of chemical processes in industries, several scientists have studied transient hydromagnetic free convection flow with higher order chemical reaction under different conditions. Das et al. [23] analysed the impact of chemical reaction on the fluid flow with uniform heat flux, near an infinite vertical plate and noticed that species concentration reduces with a surge in the rate of $1^{\text {st }}$ order chemical reaction. Seth et al. [24] examined the free convection MHD flow past an impulsively moving plate with induced magnetic field and nth order chemical reactions where the governing equations were solved numerically by a finite difference scheme. They observed that chemical reaction's order has a tendency of enhancing the species concentration. Venkateswarlu et al. [25] inspected the influence of chemical reaction on hydromagnetic natural convection Jeffrey fluid in the vicinity of a permeable moving vertical plate with the consideration of heat source.

The present study seeks to explore the effect of thermo- diffusion phenomena on the transient hydromagnetic free convection flow of an incompressible, viscous, optically thick heat radiating, chemically reactive and electrically conducting fluid near a vertical infinite plate through a porous medium with second order homogenous chemical reaction. Three varieties of nanoparticles, namely, aluminum oxide $\left(\mathrm{Al}_{2} \mathrm{O}_{3}\right)$, silver $(\mathrm{Ag})$ and titanium oxide $\left(\mathrm{TiO}_{2}\right)$ are chosen for this study. To the best of our information, no attempt has been made to undertake this kind of study. Such types of flow problem may arise in several industrial processes such as coolant application, manufacture of ceramics and glassware, nanodrug delivery, cooling of microchip, polymer production, etc.

\section{MATHEMATICAL FORMULATION}

Consider the transient magnetohydrodynamic free convection flow with the second order chemical reaction and Soret effect, of chemically reactive, optically thick heat radiating, incompressible, viscous and electrically conducting nanofluid near a vertical plate which is of infinite length and whose movement is impulsive, through a porous medium. Cartesian coordinate system is chosen to represent the geometry of the present model, such that the plate is considered to be in the positive direction of $x^{\prime}$-axis. $y^{\prime}$-axis is in a direction which is perpendicular to the plate whereas $z^{\prime}-$ axis is in the normal direction to $x^{\prime} y^{\prime}$ - plane. There is an application of an unvarying magnetic field of strength $B_{0}$ in a direction which is transversal to the motion of the plate. For $t^{\prime} \leq 0$, i.e. in the initial phase, the plate as well as the fluid, both are kept at rest and are kept at constant temperature $T_{\infty}^{\prime}$ whereas level of concentration of nanoparticles is maintained at a constant value $C_{\infty}^{\prime}$. The plate start moving impulsively in its own plane with velocity $\lambda U_{0}$ in the positive direction of $x^{\prime}$-axis at $t^{\prime}>0$, where $U_{0}$ is constant. The geometry of the present model is portrayed in Fig.1. Due to the small magnetic Reynolds number of metallic liquids, the influence of induced magnetic field has been ignored.

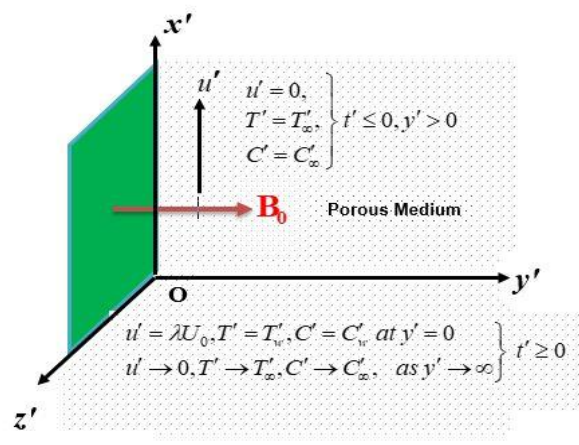

Figure 1. Geometry of the problem

Following the above made assumptions, the boundary layer equations governing the nanofluid velocity, temperature and distribution of nanoparticles, taking second order chemical reaction and Soret effect into account, under the Boussinesq approximation is given as follows:

$$
\begin{aligned}
\rho_{n f} \frac{\partial u^{\prime}}{\partial t^{\prime}}= & \mu_{n f} \frac{\partial^{2} u^{\prime}}{\partial y^{\prime 2}}-\left(\sigma_{n f} B_{0}^{2}+\frac{\mu_{n f}}{k^{\prime}}\right) u^{\prime}+g\left(\rho \beta_{C}\right)_{n f}\left(C^{\prime}-C_{\infty}^{\prime}\right) \\
& +g\left(\rho \beta_{T}\right)_{n f}\left(T^{\prime}-T_{\infty}^{\prime}\right)
\end{aligned}
$$


$\frac{\partial T^{\prime}}{\partial t^{\prime}}=\frac{k_{1 n f}}{\left(\rho c_{p}\right)_{n f}} \frac{\partial^{2} T^{\prime}}{\partial y^{\prime 2}}-\frac{1}{\left(\rho c_{p}\right)_{n f}} \frac{\partial^{2} q_{r}^{\prime}}{\partial y^{\prime 2}}$

$\frac{\partial C^{\prime}}{\partial t^{\prime}}=D_{n f} \frac{\partial^{2} C^{\prime}}{\partial y^{\prime 2}}+D_{m} \frac{\partial^{2} T^{\prime}}{\partial y^{\prime 2}}-k_{2}^{\prime}\left(C^{\prime}-C_{\infty}^{\prime}\right)^{2}$

where $u^{\prime}, T^{\prime}, C^{\prime}, \rho_{n f}, k_{1 n f}, \sigma_{n f},\left(\beta_{T}\right)_{n f},\left(\beta_{c}\right)_{n f}, \mu_{n f}, q_{r}^{\prime}, B_{0}, k^{\prime}$, $D_{M}$ and $g$, are fluid velocity, fluid temperature, species concentration, nanofluid density, thermal conductivity of nanofluid, electrical conductivity of nanofluid, thermal expansion coefficient of nanofluid, coefficient of expansion for species concentration, dynamic viscosity of nanofluid, radiative flux vector, uniform magnetic field, porous medium's permeability, thermal diffusion coefficient and acceleration due to gravity, respectively.

For nanofluid, the expressions of

$\rho_{n f},\left(\rho \beta_{T}\right)_{n f},\left(\rho \beta_{c}\right)_{n f}, \mu_{n f}, \sigma_{n f},\left(\rho c_{p}\right)_{n f}$ and $k_{n f}$ are given as:

$\rho_{n f}=(1-\psi) \rho_{f}+\psi \rho_{s},\left(\rho \beta_{T}\right)_{n f}=\psi\left(\rho \beta_{T}\right)_{s}+(1-\psi)\left(\rho \beta_{T}\right)_{f}$,

$\left(\rho \beta_{c}\right)_{n f}=\psi\left(\rho \beta_{c}\right)_{s}+(1-\psi)\left(\rho \beta_{c}\right)_{f}, \mu_{n f}=\frac{\mu_{f}}{(1-\psi)^{2.5}}$,

$\sigma_{n f}=\sigma_{f}\left(1+\frac{3(\sigma-1) \psi}{(\sigma+2)-(\sigma-1) \psi}\right)$,

$\left(\rho c_{p}\right)_{n f}=\psi\left(\rho c_{p}\right)_{s}+(1-\psi)\left(\rho c_{p}\right)_{f}$,

$\frac{k_{n f}}{k_{f}}=\frac{-2 \psi\left(k_{f}-k_{s}\right)+\left(k_{s}+2 k_{f}\right)}{\psi\left(k_{f}-k_{s}\right)+\left(k_{s}+2 k_{f}\right)}$,

where $\rho_{s}$ is density of nanoparticles, $\rho_{f}$ is the density of base fluid, $\mu_{f}$ is base fluid's viscosity, $\psi$ is volume fraction of nanoparticle, $\left(\rho c_{p}\right)_{f}$ is the heat capacitance of base fluid, $\left(\rho c_{p}\right)_{s}$ is the heat capacitance of nanoparticles, $\sigma_{s}$ is nanoparticles' electrical conductivity, $k_{s}$ is nanoparticles' thermal conductivity and $k_{f}$ is base fluid's thermal conductivity.

Following are the appropriate boundary and initial conditions:

$u^{\prime}=0, C^{\prime}=C_{\infty}^{\prime}, T^{\prime}=T_{\infty}^{\prime}$, for $y^{\prime}>0$, and $t^{\prime} \leq 0$,

$\left.\begin{array}{l}u^{\prime}=\lambda U_{0}, T^{\prime}=T_{w}^{\prime}, C^{\prime}=C_{w}^{\prime} \quad \text { at } y^{\prime}=0 \\ u^{\prime} \rightarrow 0, T^{\prime} \rightarrow T_{\infty}^{\prime}, C^{\prime} \rightarrow C_{\infty}^{\prime} \quad \text { as } y^{\prime} \rightarrow \infty\end{array}\right\} t^{\prime} \geq 0$

Table 1. Thermo-physical properties of nanoparticles and water

\begin{tabular}{|c|c|c|c|c|c|}
\hline & $\begin{array}{c}c_{p} \\
\left(\mathrm{Jg}^{-1} \mathrm{~K}^{-1}\right)\end{array}$ & $\begin{array}{c}k \\
\left(\mathrm{Wm}^{-1} \mathrm{~K}^{-1}\right)\end{array}$ & $\sigma(\mathrm{S} / \mathrm{m})$ & $\begin{array}{c}\beta \times 10^{-5} \\
\left(K^{-1}\right)\end{array}$ & $\rho\left(\mathrm{kgm}^{-3}\right)$ \\
\hline $\mathrm{Al}_{2} \mathrm{O}_{3}$ & 765 & 40 & $\begin{array}{c}1.07 \times 10^{-} \\
6\end{array}$ & 0.85 & 3970 \\
\hline $\mathrm{MoS}_{2}$ & 397.21 & 904.4 & $\begin{array}{c}2.09 \times 10^{-} \\
4\end{array}$ & 2.8424 & $5.06 \times 10^{-3}$ \\
\hline $\mathrm{Ag}$ & 235 & 429 & $6.30 \times 10^{7}$ & 1.89 & 10500 \\
\hline $\mathrm{H}_{2} \mathrm{O}$ & 4179 & 0.613 & $5.5 \times 10^{-6}$ & 21 & 997.1 \\
\hline
\end{tabular}

When the fluid is an optically thick radiating one, we can approximate the radiative flux $q_{r}^{\prime}$ by the expression proposed by the Rosseland. Therefore, according to the Rosseland approximation [26], for a medium which is considered optically thick, the radiative heat flux $q_{r}^{\prime}$ is given as:

$q_{r}^{\prime}=-\frac{4}{3}\left(\frac{\sigma^{*}}{k^{*}}\right) \frac{\partial T^{\prime 4}}{\partial y^{\prime}}$,

where $k^{*}$ is Rosseland mean absorption co-efficient and $\sigma^{*}$ is Stefan-Boltzmann constant. It is supposed that temperature difference inside boundary layer region is small enough such that the term $T^{\prime 4}$ can be presented as a linear function of temperature $T^{\prime}$. Therefore $T^{\prime 4}$ may be expanded using Taylor's expansion about $T_{\infty}^{\prime}$ and by neglecting terms of order more than and equal to two in the expansion, we get

$T^{\prime 4} \cong 4 T_{\infty}^{\prime 3} T^{\prime}-3 T_{\infty}^{\prime 4}$

Making use of (5a) and (5b), equation (2) takes the following form:

$\frac{\partial T^{\prime}}{\partial t^{\prime}}=\frac{k_{1 n f}}{\left(\rho c_{p}\right)_{n f}}\left(1+\frac{16 \sigma * T_{\infty}^{\prime 3}}{3 k k^{*}}\right) \frac{\partial^{2} T^{\prime}}{\partial y^{\prime 2}}$.

To present equations (1), (3) and (6) together with boundary and initial conditions (4a) to (4b) in dimensionless form, we use following variables which are non-dimensional in nature:

$\left.\begin{array}{l}y=y^{\prime} / U_{0} t_{0}, T=\left(T^{\prime}-T_{\infty}^{\prime}\right) /\left(T_{w}^{\prime}-T_{\infty}^{\prime}\right), u=u^{\prime} / U_{0}, \\ C=\left(C^{\prime}-C_{\infty}^{\prime}\right) /\left(C_{w}^{\prime}-C_{\infty}^{\prime}\right), t=t^{\prime} / t_{0}\end{array}\right\}$

Using non-dimensional variables given by equation (7), equations (1), (3) and (6) get converted into following dimensionless form:

$\frac{\partial u}{\partial t}=b_{1} \frac{\partial^{2} u}{\partial y^{2}}+b_{2} G r T+b_{3} G c C-M b_{4} u-b_{4} \frac{u}{K}$,

$\frac{\partial T}{\partial t}=b_{5} \frac{\partial^{2} T}{\partial y^{2}}$

$\frac{\partial C}{\partial t}=\frac{1}{S c} \frac{\partial^{2} C}{\partial y^{2}}-K r C^{2}+S r \frac{\partial^{2} T}{\partial y^{2}}$,

The dimensionless boundary and initial conditions are presented as:

$u=0, T=0, C=0$ for $t \leq 0$ and $y \geq 0$

$\left.\begin{array}{l}u=\lambda, T=1, C^{\prime}=1 \quad \text { at } y=0 \\ u \rightarrow 0, C \rightarrow 0, T \rightarrow 0 \quad \text { as } y \rightarrow 0\end{array}\right\} t \geq 0$

where $G r=\frac{v_{f} g \beta_{T f}\left(T_{w}^{\prime}-T_{\infty}^{\prime}\right)}{U_{0}{ }^{3}}$ is thermal Grashof number, $N r=$ $\frac{16 \sigma^{*} T_{\infty}^{\prime 3}}{3 k_{1 n f} f^{\prime}}$ is radiation parameter, $S c=\frac{v_{f}}{D_{n f}}$ is Schmidt number, 
$G m=\frac{v_{f} g \beta_{c f}\left(C_{w}^{\prime}-C_{\infty}^{\prime}\right)}{U_{0}{ }^{3}}$ is solutal Grashof number, $\operatorname{Pr}=\frac{\left(\mu c_{p}\right)_{f}}{k_{1 f}}$ is Prandtl number, $K r=\frac{v_{f} k_{2}\left(C_{w}^{\prime}-C_{\infty}^{\prime}\right)}{U_{0}{ }^{2}}$ is chemical reaction parameter, $M=\frac{v_{f} \sigma_{f} B_{0}^{2}}{\rho_{n f} U_{0}^{2}}$ is magnetic parameter and $S r=$ $\frac{D_{M}\left(T_{w}^{\prime}-T_{\infty}^{\prime}\right)}{v_{f}\left(C_{w}^{\prime}-C_{\infty}^{\prime}\right)}$ is Soret number.

The other parameters and variables appearing in equations (8a) to (8c) are given as:

$$
\begin{aligned}
& b_{1}=\frac{\mu_{f}}{(1-\psi)^{2.5}}, b_{2}=\frac{x_{2}}{x_{3}}, b_{3}=\frac{x_{3}}{x_{1}} \text {, } \\
& b_{4}=\frac{x_{6}}{x_{1}}, \\
& b_{5}=\frac{1}{x_{4} \operatorname{Pr}}\left(x_{5}+N r\right) \text {, }
\end{aligned}
$$$$
\text { where } x_{1}=(1-\psi)+\psi\left(\frac{\rho_{s}}{\rho_{f}}\right), x_{2}=\left[(1-\psi)+\psi \frac{\left(\rho \beta_{T}\right)_{s}}{\left(\rho \beta_{T}\right)_{f}}\right] \text {, }
$$$$
x_{3}=\left[(1-\psi)+\psi \frac{\left(\rho \beta_{c}\right)_{s}}{\left(\rho \beta_{c}\right)_{f}}\right], x_{4}=\left[(1-\psi)+\psi \frac{\left(\rho c_{p}\right)_{s}}{\left(\rho c_{p}\right)_{f}}\right] \text {, }
$$$$
x_{5}=\frac{\left(k_{s}+2 k_{f}\right)-2 \psi\left(k_{f}-k_{s}\right)}{\left(k_{s}+2 k_{f}\right)+\psi\left(k_{f}-k_{s}\right)}, \frac{1}{K}=\frac{v_{n f} \psi v}{k U_{0}^{2}} \text {, }
$$

and $x_{6}=\left(1+\frac{3(\sigma-1) \psi}{(\sigma+2)-(\sigma-1) \psi}\right)$.

\section{NUMERICAL SOLUTION}

Equations (8a) to (8c) are coupled, partial differential equations where equation $(8 \mathrm{c})$ is non-linear in nature, which therefore, cannot be solved analytically. Therefore, we solve these equations by implicit finite difference method of CrankNicolson type. This scheme corresponding to equations (8a) to (8c) with initial and boundary conditions (9a) and (9b) are applied in following manner:

The region is discretized as rectangular grid with grid lines parallel to the coordinate axes with placing $\Delta y$ and $\Delta t$ in space and time respectively. Also $\Delta y=y_{i+1}-y_{i}$ and $\Delta t=t_{j+1}-$ $t_{j}$. With the known values of $C, T$ and $u$ at a time $t=j$, the finite difference equation corresponding to (8b) at each interval nodal point on a specific $j$ - level creates a system of tridiagonal equations. This system is solved by the use of Thomas algorithm, as proposed by Carnahan et al. [27]. In this way, the $T$ values are determined at every nodal point at $(j+1)^{\text {th }}$ time step. In the same manner, the $C$ values are determined from equation (8c) using the $T$ values at $(j+$ $1)^{\text {th }}$ time step of equation (8c). Finally, with the use of $C$ and $T$ values at $(j+1)^{\text {th }}$ time step in equation (8a), the value of $u$ at $(j+1)^{\text {th }}$ time step are obtained in similar fashion. This procedure is continued to obtain the solution until desired time $t$. Therefore, the values of $T, C$ and $u$ are determined at all points of the rectangular expanse for the desired time level. There is a truncation error of order $O\left\{(\Delta y)^{2}+(\Delta t)^{2}\right\}$ in the Crank-Nicolson scheme which diminishes as $\Delta y$ and $\Delta t$ approach to zero, which justifies the consistency (Antia [28]). Consistency and stability together guarantee the convergence of scheme.

\section{WALL VELOCITY GRADIENT, WALL TEMPERATURE GRADIENT AND CONCENTRATION GRADIENT IN THE VICINITY OF THE PLATE}

Expression for wall velocity gradient $\tau$, wall temperature gradient $\mathrm{Nu}$ and concentration gradient in the vicinity of the plate, $S_{h}$ are calculated from equations (10) to (12) respectively and are presented as follows:

$\tau=\left.\frac{\partial u}{\partial y}\right|_{y=0}$

$N u=\left.\frac{\partial T}{\partial y}\right|_{y=0}$

$S_{h}=\left.\frac{\partial C}{\partial y}\right|_{y=0}$

\section{RESULTS AND DISCUSSION}

To highlight the impact of several physical parameters which characterize the features of the flow, extensive numerical calculations have been performed for nanofluid velocity, temperature and species concentration together with wall velocity gradient, wall temperature gradient and concentration gradient near the plate, to get an insight of the physics involved in the flow regime.

Numerical results which are demonstrated in Figures 2-11, have been scrutinized in detail by giving numerical values to physical parameters. Throughout the study, we take the following values of non-dimensional numbers: $G r=5, G c=4$, $N r=4, M=3, S r=2, K r=3, \varphi=0.15, \gamma=1, \operatorname{Pr}=0.71$. $S c=0.22, \lambda=1$ and $K=2$, unless stated otherwise.

The variation in the nanofluid velocity versus the boundary layer coordinate $y$, under the influence of various parameters are depicted graphically in Figures 2 to 6. Figures 2 and 3 portray the influence of $G r$ and $G c$ on nanofluid velocity $u$, respectively. It is conceived form these Figures that nanofluid velocity $u$ increases on increasing the values of $G r$ and $G c$. Since, an increase in $G r$ and $G c$ means an increase in the strengths of thermal and solutal buoyancy forces, respectively. This infers that solutal as well as thermal buoyancy forces tend to speed-up the fluid velocity.

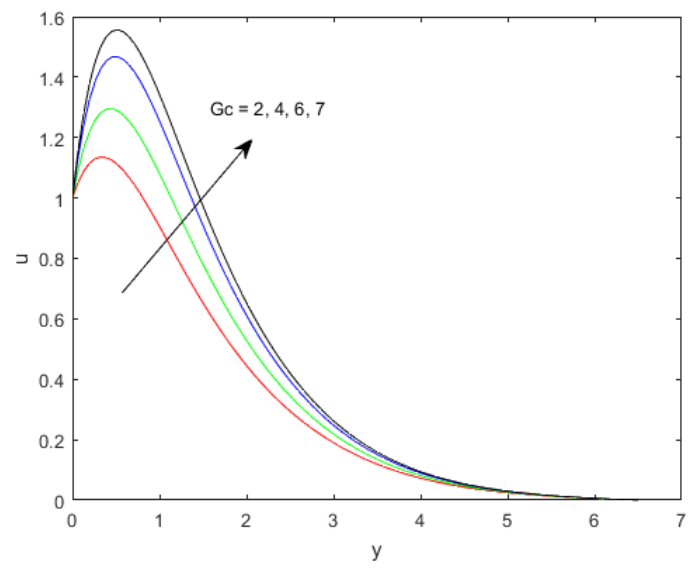

Figure 2. Velocity profiles for $G c$ 


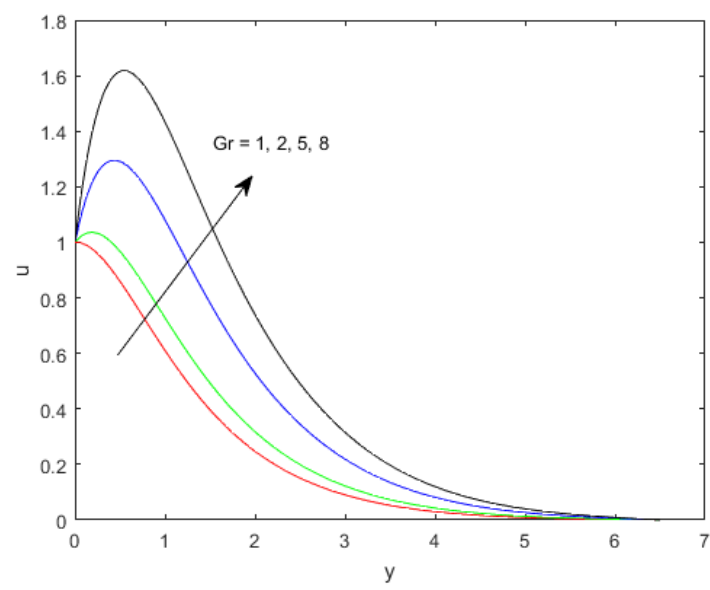

Figure 3. Velocity profiles for $\mathrm{Gr}$

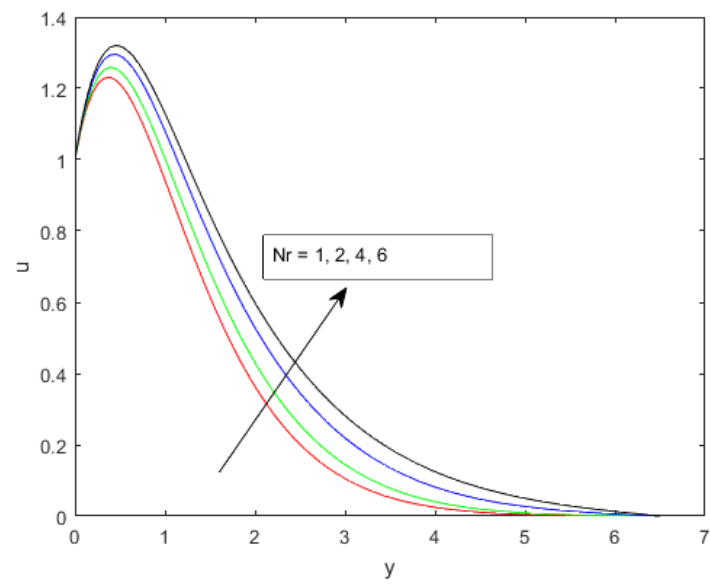

Figure 4. Velocity profiles for $\mathrm{Nr}$

It is perceived from Figure 4 that, nanofluid velocity $u$ is getting accelerated with the growing value of radiation parameter $N r$. The reason behind such behavior of nanofluid velocity is that an intensification in radiation parameter outcomes in the increase of fluid temperature, and therefore thermal buoyancy force becomes stronger with amplified thermal radiation. This enhanced thermal buoyancy force results in quicker fluid motion.

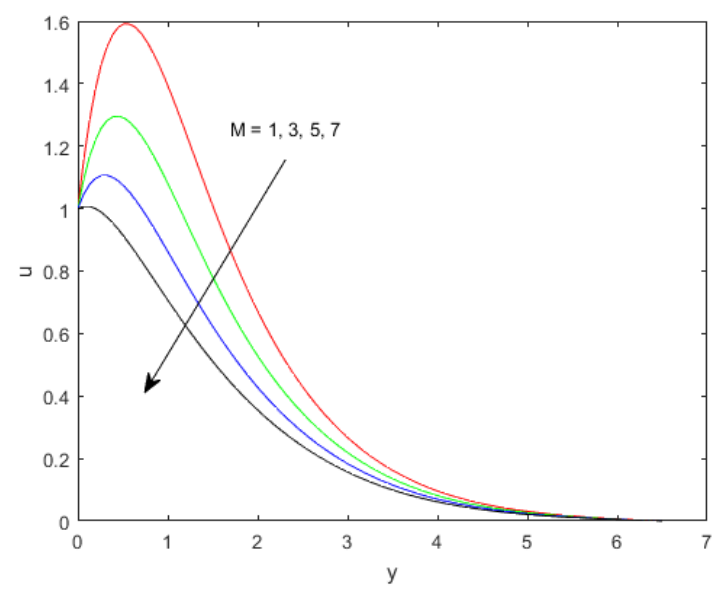

Figure 5. Velocity profiles for $M$

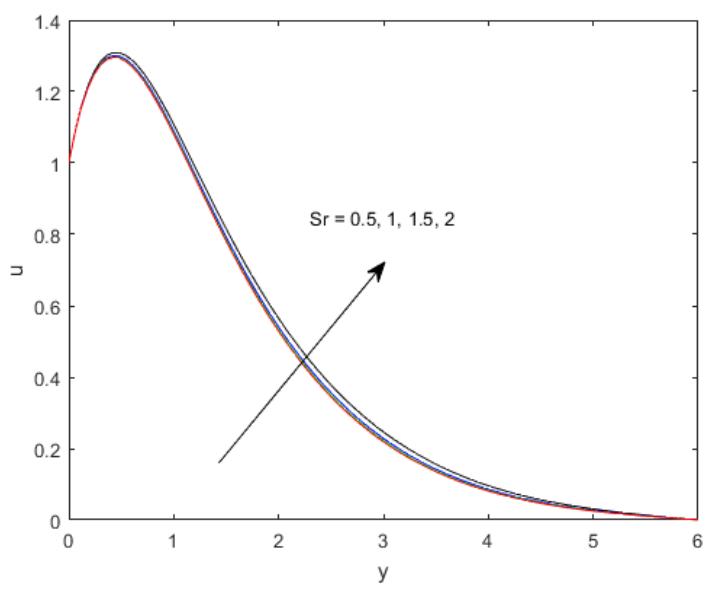

Figure 6. Velocity profiles for $\mathrm{Sr}$

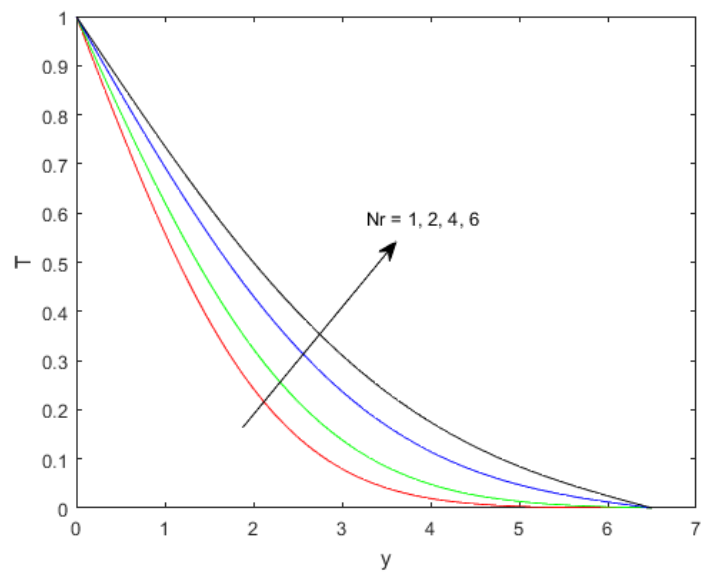

Figure 7. Temperature profiles for $\mathrm{Nr}$

It is noticed from Figure 5 that $u$ is getting slowed down on strengthening the magnetic field. This fact is justified since the presence of magnetic field produces an opposing force, termed as Lorentz force, which works in a direction, reverse to the fluid course and has the tendency to resist the flow and hence a decelerated fluid motion is observed in this case.

It is perceived from Figure 6 that nanofluid velocity $u$ is increased with the growing value of Soret number $\mathrm{Sr}$. This is in agreement with the fact that a rise in the value of Soret number enhances the solutal buoyancy force which results in accelerated fluid motion.

Figure 7 depicts, how the fluid temperature $T(y, t)$ is affected by the radiation parameter $\mathrm{Nr}$. One can observe from this figure that temperature of nanofluid rises with the growing value of radiation parameter $\mathrm{Nr}$.

The influence of nanoparticle volume fraction $\varphi$ on temperature is depicted in the Figure 8, in which temperature is enhanced as the nanoparticle volume fraction is increased.

To demonstrate the characteristics of species concentration against chemical reaction, thermal-diffusion and mass diffusion parameters, the numerical solutions of species concentration $C(y, t)$ are obtained and are revealed in form of graphs in Figures 9 to 11. Figure 9 demonstrates concentration profile against Schmidt number $S c$. It is noticed that species concentration $C$ decreases on increasing the value of $S c$. This phenomenon is in good agreement with the fact that a rise in $S c$ leads to a decrement in the molecular diffusion. 


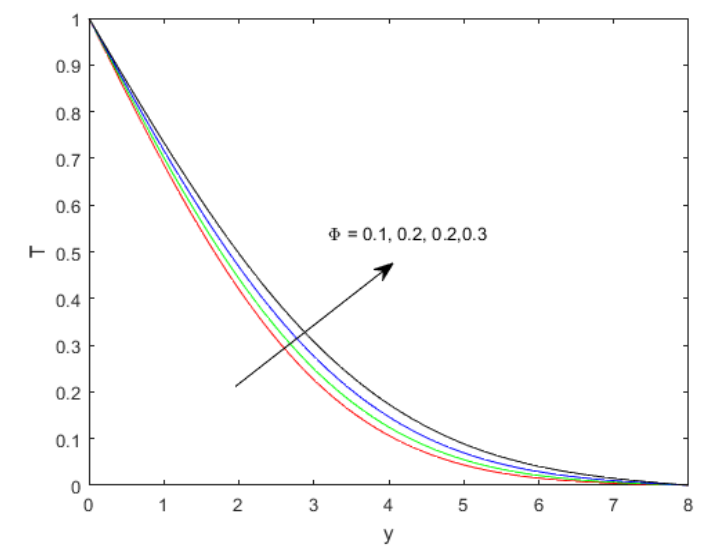

Figure 8. Temperature profiles for $\varphi$

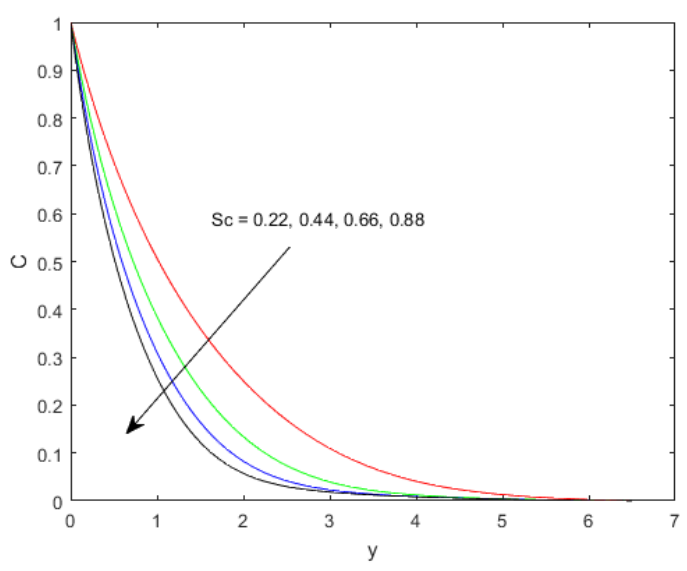

Figure 9. Concentration profiles for $S c$

Highlights of the influence of Soret number $\mathrm{Sr}$ on concentration profile is represented in Figure 10. Concentration of species grows on increasing the value of $\mathrm{Sr}$. This infers that Soret number tends to enhance species concentration.

It is evident from Figure 11 that concentration of species diminishes with the growing value of chemical reaction parameter $K r$. The central reason behind this fact is that destructive kind of chemical reaction leads to a decline in the species concentration by virtue of which, the buoyancy effect due to concentration gradient gets nullified.

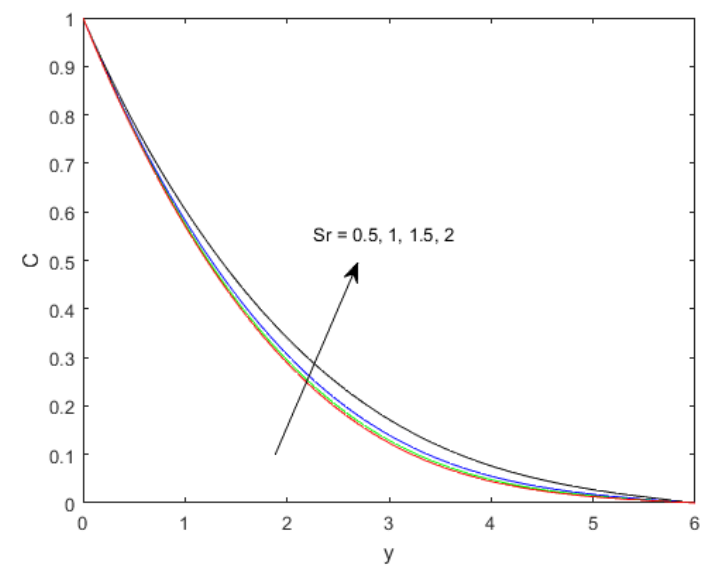

Figure 10. Concentration profiles for $\mathrm{Sr}$

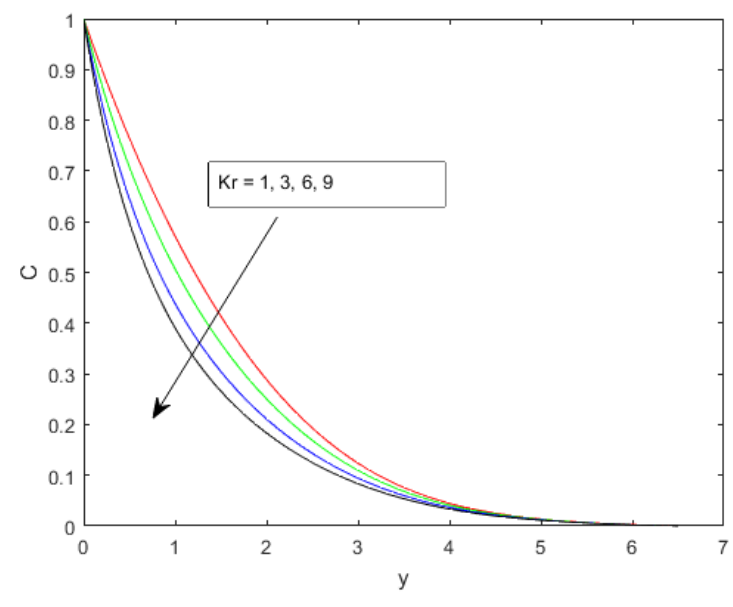

Figure 11. Concentration profiles for $\mathrm{Kr}$

The wall velocity gradient which represent skin friction at the plate together with wall temperature gradient i.e. Nusselt number and gradient in the concentration in the vicinity of the plate, for various parameters of interest are depicted in Tables 2-4. In these tables, we notice that there is a reduction in the coefficient of skin friction with a rise in the values of thermal Grashof number, solutal Grashof number, Soret number, radiation parameter while it increases for magnetic parameter. Rate of heat transfer and rate of mass transfer increases for chemical reaction parameter and Schmidt number while these quantities decrease with radiation parameter and Soret number.

\section{CONCLUSION}

The current study focuses on the theoretical investigation of Soret effect on transient magnetohydrodynamic natural convection flow of an optically thick heat radiating, and chemically reactive nanofluid near a vertical infinite plate through a porous medium in the existence of second order homogeneous chemical reaction. The noteworthy findings of the boundary layer flow of the present study are briefed as follows:

- Throughout the boundary layer region, thermal and mass buoyancy forces, thermal radiation and thermodiffusion effect tend to accelerate nanofluid velocity but completely opposite effect is observed for magnetic field and chemical reaction parameters.

- Tendency of thermal radiation is to enhance the fluid temperature in the boundary layer region.

- Mass diffusion and chemical reaction effects tend to reduce the species concentration of the nanofluid whereas a totally reverse effect is noticed on it for thermo-diffusion effect.

- We see a reduction in the Coefficient of Skin friction with a rise in the values of thermal Grashof number, solutal Grashof number, Soret number, radiation parameter while it increases for magnetic parameter. Rate of heat transfer and rate of mass transfer increases for chemical reaction parameter and Schmidt number while these quantities decrease with radiation parameter and Soret number. 
Table 2. Skin friction coefficient $(\tau)$

\begin{tabular}{|c|c|c|c|c|c|c|}
\hline$G r$ & $G c$ & $M$ & $K r$ & $S r$ & $N r$ & $\tau$ \\
\hline $\begin{array}{l}2 \\
3 \\
5\end{array}$ & 4 & 3 & 3 & 0.5 & 4 & $\begin{array}{l}1.2996 \\
0.6132 \\
0.2699\end{array}$ \\
\hline 5 & $\begin{array}{l}2 \\
4 \\
6 \\
\end{array}$ & 3 & 3 & 0.5 & 4 & $\begin{array}{c}1.1546 \\
0.2699 \\
0.1257\end{array}$ \\
\hline 5 & 4 & $\begin{array}{l}1 \\
3 \\
5\end{array}$ & 3 & 0.5 & 4 & $\begin{array}{l}0.1983 \\
0.2699 \\
0.3416\end{array}$ \\
\hline 5 & 4 & 3 & $\begin{array}{l}1 \\
3 \\
6 \\
\end{array}$ & 0.5 & 4 & $\begin{array}{l}0.7867 \\
0.2699 \\
0.1239 \\
\end{array}$ \\
\hline 5 & 4 & 3 & 3 & $\begin{array}{l}0.2 \\
0.5 \\
0.8 \\
\end{array}$ & 4 & $\begin{array}{l}0.5543 \\
0.2699 \\
0.1126 \\
\end{array}$ \\
\hline 5 & 4 & 3 & 3 & 0.5 & $\begin{array}{l}1 \\
2 \\
4\end{array}$ & $\begin{array}{l}0.9657 \\
0.2699 \\
0.0219\end{array}$ \\
\hline
\end{tabular}

Table 3. Rate of heat transfer $(N u)$

\begin{tabular}{|c|c|c|}
\hline$N r$ & $K r$ & $-N u$ \\
\hline 1 & & 0.7845 \\
2 & 0.3 & 0.4586 \\
4 & & 0.2451 \\
\hline & 1 & 0.1723 \\
& 3 & 0.3984 \\
& 6 & 0.5126 \\
\hline
\end{tabular}

Table 4. Rate of mass transfer $\left(S_{h}\right)$

\begin{tabular}{|c|c|c|c|}
\hline$S c$ & $K r$ & $S r$ & $-S_{h}$ \\
\hline 0.22 & & & 0.1838 \\
0.44 & 3 & 0.5 & 0.3746 \\
0.66 & & & 0.5186 \\
\hline & 1 & & 0.0914 \\
0.22 & 3 & 0.5 & 0.1838 \\
& 6 & & 0.2435 \\
\hline & & 0.2 & 0.4923 \\
0.22 & 3 & 0.5 & 0.1838 \\
& & 0.8 & 0.0547 \\
\hline
\end{tabular}

\section{REFERENCES}

[1] Choi SUS. (1995). Enhancing thermal conductivity of fluids with nanoparticles. Proceedings of the ASME International Mechanical Engineering Congress and Exposition 66: 99-105.

[2] Selvakumar RD, Dhinakaran S. (2016). Effective viscosity of nanofluids: a modified Krieger-Dougherty model based on particle size distribution (PSD) analysis. Journal of Molecular Liquids 225: 20-27. https://doi.org/10.1016/j.molliq.2016.10.137

[3] Heris SZ, Esfahany MN, Etemad SG. (2007). Experimental investigation of convective heat transfer of $\mathrm{Al}_{2} \mathrm{O}_{3}$ /water nanofluid in circular tube. J. Heat Fluid Flow 28: 203-210 https://doi.org/10.1016/j.ijheatfluidflow.2006.05.001

[4] Fotukian SM, Nasr Esfahany M. (2010). Experimental study of turbulent convective heat transfer and pressure drop of dilute $\mathrm{CuO} /$ water nanofluid inside a circular tube. I. J. Commun. Heat Mass Transf. 37: 214-219. https://doi.org/10.1016/j.icheatmasstransfer.2009.10.00 3

[5] Turkyilmazoglu M. (2012). Exact analytical solutions for heat and mass transfer of MHD slip flow in nanofluids. Chem. Eng. Sci. 84: 182-187. https://doi.org/10.1016/j.ces.2012.08.029

[6] Sheikholeslami M, Bandpy M.G, Ellahi R, Zeeshan A. (2014). Simulation of MHD $\mathrm{CuO}$ water nanofluid flow and convective heat transfer considering Lorentz forces. J. Magn. Magn. Mater. 369: 69-80.

[7] Sandeep N, Reddy MG. (2017). Heat transfer of nonlinear radiative magnetohydrodynamic $\mathrm{Cu}$-water nanofluid flow over two different geometries. Journal of Molecular Liquids 225: 87-94. https://doi.org/10.1016/j.molliq.2016.11.026

[8] Takhar HS, Gorla RSR, Soundalgekar VM. (1996). Short communication radiation effects on MHD free convection flow of a gas past a semi-infinite vertical plate. Int. J. Numer. Methods Heat Fluid Flow 6: 77-83. https://doi.org/10.1108/09615539610113118

[9] Das S, Jana RN. (2015). Natural convective magnetonanofluid flow and radiative heat transfer past a moving vertical plate. Alexandria Engineering Journal 54: 5-64. https://doi.org/10.1016/j.aej.2015.01.001

[10] Ogulu A, Makinde OD. (2008). Unsteady hydromagnetic free convection flow of a dissipative and radiating fluid past a vertical plate with constant heat flux. Chem. Eng. Comm. 196: 454-462. https://doi.org/10.1080/00986440802484531

[11] Mahmoud MAA. (2009). Thermal radiation effect on unsteady MHD free convection flow past a vertical plate with temperature dependent viscosity. Canad. J. Chem. Eng. 87: 47-52. https://doi.org/10.1002/cjce.20135

[12] Das S, Jana RN, Chamkha AJ. (2015). Unsteady free convection flow past a vertical plate with heat and mass fluxes in the presence of thermal radiation. Journal of Applied Fluid Mechanics 8: 845-854. https://doi.org/10.18869/acadpub.jafm.73.238.23265

[13] Seth GS, Tripathi R, Sharma R. (2015). Natural convection flow past an exponentially accelerated vertical ramped temperature plate with hall effects and heat absorption. International Journal of Heat and Technology 33: 139-144. https://doi.org/10.18280/ijht.330321

[14] Seth GS, Kumbhakar B, Sarkar S. (2014). Unsteady hydromagnetic natural convection flow with heat and mass transfer of a thermally radiating and chemically reactive fluid past a vertical plate with Newtonian heating and time dependent free-stream. International Journal of Heat and Technology 32: 87-94.

[15] Ahmed N. (2012). Heat and mass transfer in Hartmann flow with Soret effect in presence of a constant heat source. Turk. J. Phys. 36: 446-460. https://doi.org/10.3906/fiz-1109-20

[16] Hussanan A, Salleh MZ, Khan I, Tahar RM, Ismail Z. (2015). Soret effects on unsteady magnetohydrodynamic mixed convection heat-and-mass-transfer flow in a porous medium with Newtonian heating. Maejo Int. J. Sci. Technol. 9: 24-245. https://doi.org/10.14456/mijst.2015.17

[17] Seth GS, Kumbhakar B, Sarkar S. (2015). Soret and Hall effects on unsteady MHD free convection flow of radiating and chemically reactive fluid past a moving vertical plate with ramped temperature in rotating system. 
Int. J. of Engg, Sci. and Tech. 7: 94-108. https://doi.org/10.4314/ijest.v7i2.8

[18] Zhao J, Zheng L, Zhang X. Liu F. (2016). Convection heat and mass transfer of fractional MHD Maxwell fluid in a porous medium with Soret and Dufour effects. Int. J. of Heat and Mass Transf. 103: 203-210. https://doi.org/10.1016/j.ijheatmasstransfer.2016.07.057

[19] Reddy PS, Chamkha AJ. (2016). Soret and Dufour effects on unsteady MHD heat and mass transfer from a permeable stretching sheet with thermophoresis and nonuniform heat generation/absorption. Journal of Applied Fluid Mechanics 9: 2443-2455. https://doi.org/10.18869/acadpub.jafm.68.236.25171

[20] Saritha K, Rajasekhar MN, Reddy BS. (2017). Heat and mass transfer of laminar boundary layer flow of nonNewtonian power law fluid past a porous flat plate with Soret and DuFour effects. Physical Science International Journal 11: 1-13. https://doi.org/10.9734/PSIJ/2016/26957

[21] Bég OA, Bakier AY, Prasad VR. (2009). Numerical study of free convection magnetohydrodynamic heat and mass transfer from a stretching surface to a saturated porous medium with Soret and Dufour effects. Computational Materials Science 46: 57-65. https://doi.org/10.1016/j.commatsci.2009.02.004

[22] Abdel-wahed MS, Abdel-AAL SM. (2016). Soret and DuFour effects on MHD stagnation point flow and heat transfer impinging on stretching sheet with Chemical reaction and transpiration. European Journal of Scientific Research 137: 63-73.

[23] Das UN, Deka R, Soundalgekar VM. (1994). Effects of mass transfer on flow past an impulsively started infinite vertical plate with constant heat flux and chemical reaction. Forschung im Ingenieurwesen 60: 284-287.

[24] Seth GS, Sarkar S. (2015). Hydromagnetic natural convection flow with induced magnetic field and $n^{\text {th }}$ order chemical reaction of a heat absorbing fluid past an impulsively moving vertical plate with ramped temperature. Bulgarian Chemical Communications 47: 66-79. https://doi.org/10.1016/j.joems.2014.03.006

[25] Venkateswarlu S, Kumar GV, Durga Prasad P, Varma SVK. (2017). Effect of Dufour number and chemical reaction on MHD free convection flow of Jeffrey fluid past a vertical permeable moving plate. Int. J. of Pure and App. Math. 113: 169-177.

[26] Rosseland S. (1931). Astrophysik und atom-theoretische Grundlagen, Springer-Verlag, Berlin.
[27] Carnahan B, Luther HA, Wilkes JO. (1969). Applied Numerical Methods, New York, John Wiley.

[28] Antia H.M. (1991). Numerical methods for scientists and engineers. New Delhi: Tata McGraw-Hill Publishing Co Ltd.

\section{NOMENCLATURE}

$\begin{array}{ll}M & \text { Magnetic parameter } \\ S c & \text { Schmidt number } \\ g & \text { Acceleration due to gravity } \\ B_{0} & \text { Uniform magnetic field } \\ \operatorname{Pr} & \text { Prandtl number } \\ K r & \text { Chemical reaction parameter } \\ N r & \text { Radiation parameter } \\ S r & \text { Soret number } \\ G r & \text { Thermal Grashof number } \\ G m & \text { Solutal Grashof number } \\ k^{\prime} & \text { Permeability of porous medium } \\ k^{*} & \text { Rosseland mean absorption co-efficient } \\ \sigma^{*} & \text { Stefan-Boltzmann constant } \\ u^{\prime} & \text { Fluid velocity } \\ T^{\prime} & \text { Fluid Temperature } \\ C^{\prime} & \text { Species concentration }\end{array}$

\section{Greek symbols}

$\begin{array}{ll}\rho_{f} & \text { Density of base fluid } \\ \rho_{n f} & \text { Density of nanofluid } \\ \mu_{n f} & \text { Dynamic viscosity of nanofluid } \\ \mu_{f} & \text { Dynamic viscosity of base fluid } \\ \sigma_{n f} & \text { Electrical conductivity of nanofluid } \\ \sigma_{f} & \text { Electrical conductivity of base fluid } \\ \psi & \text { Nanoparticle volume fraction }\end{array}$

\section{Subscripts}

$\begin{array}{ll}s & \text { Nanoparticle } \\ f & \text { Base fluid } \\ n f & \text { Nanofluid }\end{array}$

